\title{
PC based Monitoring of Human Pulse Signal using LabVIEW
}

\author{
S.Anu Roopa Devi ${ }^{1}$, R.Keerthana ${ }^{2}$, P.Mahalakshmi ${ }^{3}$, R.Harisudhan ${ }^{4}$, S.Rathna Prabha ${ }^{5}$ \\ Student, Instrumentation and Control Engineering, Saranathan College of Engineering, Trichy, India ${ }^{1,2,3,4}$ \\ Assistant Professor, Instrumentation and Control Engineering, Saranathan College of Engineering, Trichy, India ${ }^{5}$
}

\begin{abstract}
PC based monitoring of human pulse signal monitors the pulse of a human body. This method of monitoring human pulse signal is implemented using LabVIEW interconnected with myRIO, based on the pulse of human body the pulse is sensed using XD58C (AMPED). This method of sensing the pulse signal is an indirect method. Using this method the abnormality of a person can be determined.
\end{abstract}

Keywords: Pulse, XD58C (AMPED), Pulse sensor, LabVIEW, myRIO.

\section{INTRODUCTION}

Those days in hospitals the pulse of human body is the wavelength is of 609NM. It consists of totally 3 Pins, determined only by keeping the hands on wrist, in which it the red coloured pin is for supply voltage, the white will not give a correct determination of pulse of a human coloured pin is for output and the black coloured pin is for body and in ICU's a nurse will not always be with each ground. and every patient. In this paper, therefore in order to avoid these kind of situations "PC BASED MONITORING OF PULSE SIGNAL USING LabVIEW" is implemented in which the pulse of human body is determined using a sensor, which is a non-invasive method.

\section{BLOCK DIAGRAM}

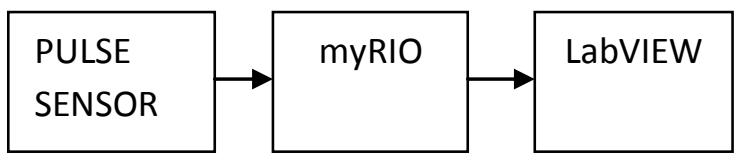

The human pulse signal is attained directly using a sensor named XD58C(AMPED). XD58C (AMPED) sensor is the one in which it has a noise cancellation circuit inbuilt. It works on the basis on which similar to an optical sensor.

The signal is obtained directly from the sensor in which it is being interfaced with the help of myRIO and then it is being implemented in LabVIEW program.

The pulse is the input given to the myRIO where the LabVIEW is being programmed according to the myRIO used. Then the output of a human pulse is monitored.

\section{HARDWARE DESCRIPTION} PULSE SENSOR XD58C (AMPED)

Fig 1 shows the pulse sensor. The pulse sensor can be worn on the finger or the earlobe and also on the wrist.

It also has an open-source app program, used for real time. Heart rate displayed by the graph. A heart rate sensor integrated optical amplifier and noise elimination circuit.

The colour of the sensor is of red and the material being made is of FR4, the power supply voltage being given is of $3 \mathrm{~V} / 5 \mathrm{~V}$. The magnification for the sensor is of 330 and

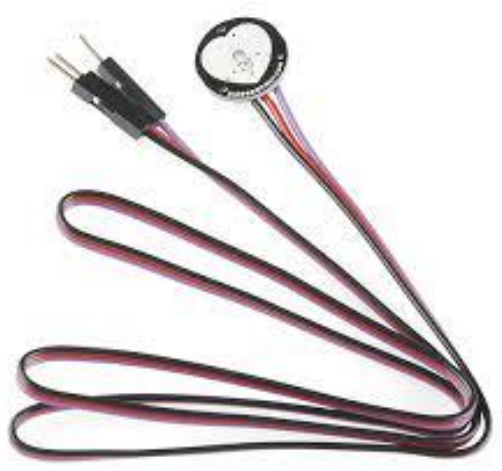

Fig 1- XD58C (AMPED) PULSE SENSOR

myRIO:-

Fig 2 shows the myRIO being used for interfacing the sensor.

NI myRIO is an embedded hardware device which is an industry proven technology which allows real and complex engineering systems into more quick and affordable.

It is a real time processing and Xilinx FPGA customizable I/O. It can be used in mechatronics, embedded systems, robotics etc,.

The program should be implemented in LabVIEW and deployed in myRIO and then connected with the third party sensors.

In this system NI myRIO is being used to get the signal from the sensor and to give it to the LabVIEW platform and to obtain the waveform. 


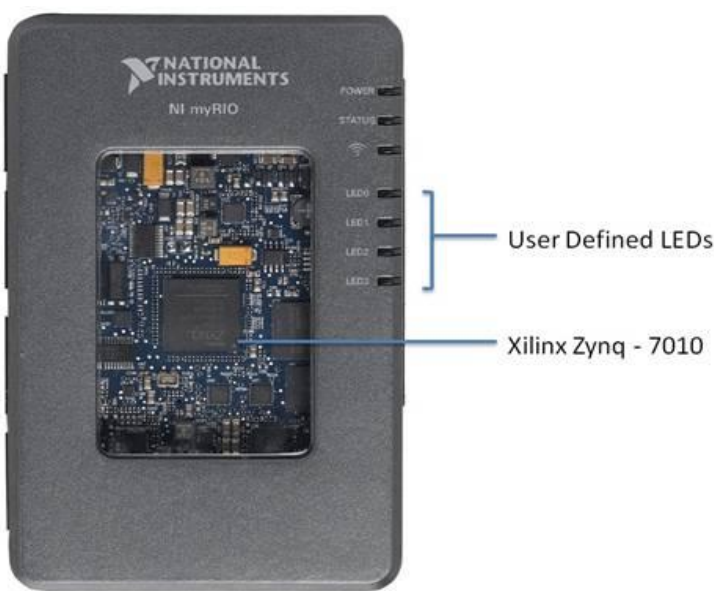

Fig 2-myRIO

\section{SOFTWARE DESCRIPTION}

LabVIEW is software in which it is being used as a platform in this system. In which the simulation can be done for the pulse sensor and the simulation will also give the value of the pulse obtained and also to show whether the pulse being taken by the sensor is normal or abnormal in which it is easy to identify whether the person is in normal or in abnormal state.

\section{SIMULATION}

The pulse sensor is the one in which it is being connected to the bread board then the sensor is being placed over the wrist of the left hand. In which it is being interfaced to the myRIO. And the LabVIEW program is being done for simulation. Fig 3 shows the simulation of pulse sensor. The simulation for pulse sensor is being done in which the normal and abnormal condition for a patient can be identified. The condition is being done depending on the pulse of a normal person. The normal person pulse is of about $70-80$ pulse per minute.

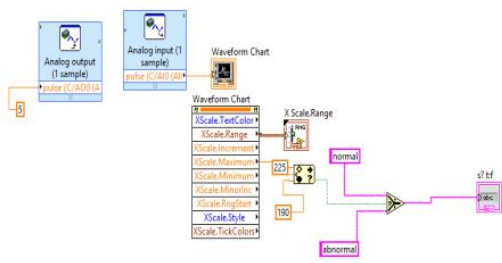

Fig 3- Simulation of pulse sensor

\section{EXECUTION}

The pulse sensor execution is being done by running the simulation of the pulse sensor. The execution for both normal and abnormal person can be executed using LabVIEW. The sensor is being placed over the wrist of the left hand in which the program is run and in the front panel the obtained result is shown as in abnormal condition in which it exceeds of about the normal pulse rate. The obtained result can also be in the form in which the normal condition can also be detected. The front panel shown below is the normal condition of human pulse. The execution of pulse sensor is as shown in fig 4(a) and 4(b).

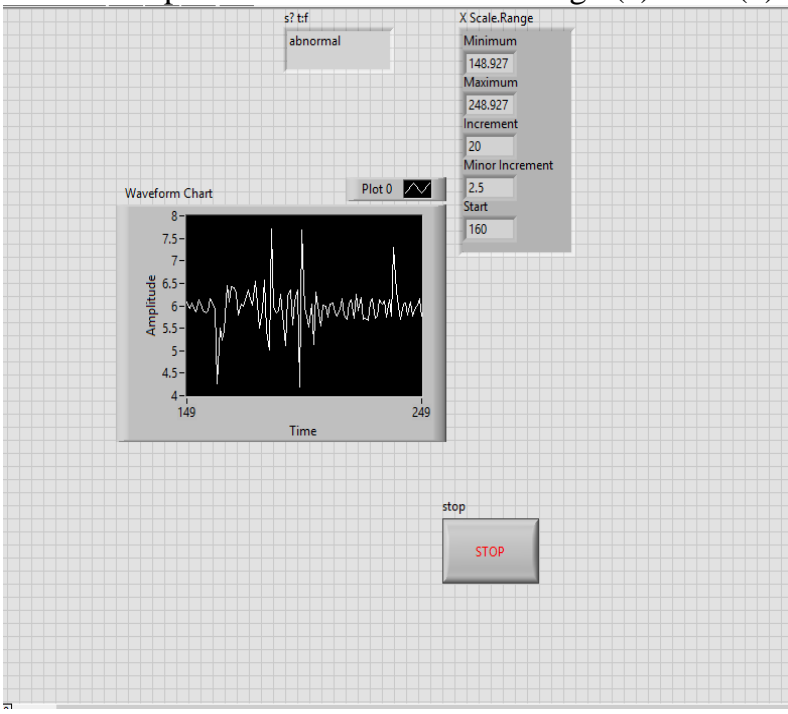

Fig 4(a)- Execution of pulse sensor (abnormal condition)

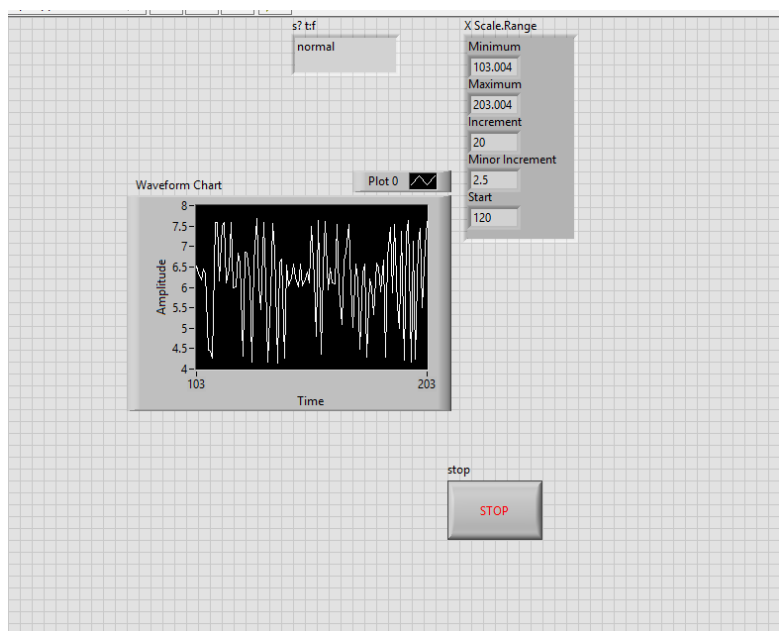

Fig 4(b)- Execution of pulse sensor (normal condition)

\section{CONCLUSION}

The pulse of the human being is being detected and the normal and abnormal condition of the person is also being detected using LabVIEW program. The normal and abnormal status of the person is being detected using the graph being displayed and the pulse being obtained.

\section{REFERENCES}

1. LabVIEW and WEB server based server based human monitoring system N.P. JOSHI and P.V.PATEL

2. Implementation of LabVIEW based packet logger for zigbee network B. UDAYA KUMAR, B.VENKAT and Dr.D.S. MURTHY. 